$\xi=-1$

\title{
Rate of Perceived Exertion of Potential Avionics Male Students and Proposed Exercises to Decrease Strain
}

\author{
Mohd Harridon* \\ Universiti Kuala Lumpur \\ *Corresponding author E-mail: mdharridon@unikl.edu.my
}

\begin{abstract}
As a student in Aviation, particularly in the field of Avionics, one has to actuate real life practical in real life aircraft. In the field of Avionics, the electrical and electronic racks are usually located in confined and constricted spaces in the cargo bay of the aircraft. To actuate works upon these spaces requires the student to crawl and be in physically stressful position for a predetermined period. If the student could not withstand these situations, then their practical workmanship would be poor. Thus, potential student in Avionics should be fit in order to perform well during these practical sessions. This paper collected the Rate of Perceived Exertion (RPE) of high school students that aspire to be Avionics students after graduating from high school. The RPE showed whether these students could withstand or not the practical in Avionics and the results showed that high school students that are fit felt less exertion, thus more likely to perform well in their practical. The results also showed that high school students that are not fit felt more exertion thus more likely to perform poorly in their practical. This paper also laid out proposed physical exercises to decrease the strain or exertion felt.
\end{abstract}

Keywords: Rate of Perceived Exertion; Avionics Rack; Physical Exercise; Avionics Practical; Physical Exertion

\section{Introduction}

Safety is gravely vital in the aviation industry. Tremendous amount of works are exerted while maintaining and fixing aircrafts which is a norm in aircraft maintenance. Workers that are in good physical condition and not subjected to arduous rigid conditions would produce high quality results maintaining the aircraft. Poor outputs pertaining to maintenance would somehow affect the safety of the aircraft and could lead to maladies, incidents, and accidents.

Federal Aviation Administration (FAA) had authored and produced a textbook or handbook called Aircraft Maintenance Textbook which indicated that $80 \%$ of accidents in aviation were cause by human factors [1]. The handbook boldly reiterated that "not conducive" working environment or condition contributes to duress which is also a component of human factors [2]. The nonconducive environments, for example tight and cramp spaces are norms when fixing aircrafts which induces physical stresses upon workers, but these stresses can be decreased if the workers are fit. A physically fit worker with optimum mass of muscle (legs are good example) and body weight that is healthy could go through kneeling, for example approximately 30 seconds, to repair aircraft components at cramp spaces.

For this project we had simulated to our respondents two actual working scenarios that induce physical stress upon aircraft workers of maintenance. Our sample consists of respondents who are male students of a selected High School in Malaysia and all of these respondents aspire to be students in the Avionics Field after graduating from High School. These students, in their future course of the avionics maintenance programme, would have to go through numerous practical (tight and open spaces) in fixing or maintaining avionics of the aircraft. Thus, we had set up the two working conditions that were mentioned earlier and the respond- ents were asked to perform tasks in these two working conditions and their Rate of Perceived Exertions (RPE) were recorded. This paper recorded their RPE that shows the degree of their duress, actuated synthesis on the RPE, and bestowed sufficient physical regime to bring down the levels of RPE. The results and analyses are significant as we could identify upfront the problems that the students would face when going through the avionics practical in their future endeavor. The recommended physical exercise would aid these graduating High School students in preparing themselves for the future Avionics Course.

\section{Literature Review of Rate of Perceived Ex- ertion (RPE)}

The intensity of physical activities could be measured by using RPE as indicated by the United States Health Department [3]. In accordance to the fitness level of the individual and the intensity of the bodily movement or work actuated, one would feel high or low physical exertion where this feeling is called Perceived Exertion. The feeling comprises the rate of the heartbeat, perspiration, muscle fatigue, and the rate of breathing.

RPE is subjective since it is based upon one's perception. Even though it is subjective, it is still adequate to be used as a measurement tool to measure one's physical capability as stated by the US Health Department. Gunnar Borg from the University of Stockholm had developed RPE. Borg invented a scale where the intention was to quantify one's strain or aches [4]. Regardless of gender age, race, and others, the scale is a good medium for comparison.

The scale is sectioned into several categories which are "Nothing at all", "Very very weak", "Very weak", "Weak", "Moderate", "Somewhat strong", "Strong", "Very Strong", and "Very very strong" but somehow Borg conceded that there is a minor drawback in actuating comparison between individuals but this does 
not affect the overall efficiency of the scale. Several variations exist with regards to the usage of the terminologies to section off the scale, but the basis is similar throughout. In this paper we are using the RPE scale that was based upon the Cleveland Clinic in the United States where the scale has a range from 0 till 10 where 0 being the easiest and 10 being the hardest.

Breathing difficulties in a person could also be rated using the scale as stated by Borg. Based upon the heuristic of the author, maintenance workers that are subjected to intense working conditions such as cramp spaces do experience minor changes in their rate of breathing, thus interestingly could be measured using the scale. The stress level of an employee is imperative to be measured as stated in the documentation or finding by Kane who is a personnel of the United States Air Force and measuring via perception, through RPE, is a sufficient way to observe or measure this stress level where this stress level is in tandem with the working conditions [5]. Kane also advised workers to physically prepare themselves via conditioning exercise in order for them to withstand extreme working conditions and along the way reduce their level of stress and hence increase safety in aviation [5].

\section{Methodology}

Figure 1 shows the methodology of this project. 50 respondents which are students from a High School in Malaysia were selected. The selected respondents were males (all of them) and were selected based on the fact that they have great interest to pursue a course in Avionics after graduating from High School. In the United States, women only comprise $2.3 \%$ of the total certified aircraft mechanic workforce, as stated by Clark in his report [6]. Hence the majority in the first line aviation maintenance would be males and we are interested in the majority, thus confining this project to only male respondents. The quality of work actuated by the male workers in relation to their fitness and workplace conditions (rigid or strainful) is of the interest of the author.

The author had selected 2 aircraft maintenance activities which are usually performed at tight spaces. These 2 tight spaces are usually transversed or converged upon by the avionics workers since the rack of the avionics is usually placed in the aircraft's cargo bay and this bay is normally compact and cramp. At the rack the workers usually kneel or sit to perform tasks on the rack. Hence within this project 2 scenarios were utilized which were: "Kneeling for 8 Seconds" (Scenario 1) \& "Crawling a Distance of $2 \mathrm{Me}$ ters" (Scenario 2).

In the Boeing 727, one has to transverse or crawl for 2 meters (exact length) to reach or get hold of the avionics rack [7]. The length of the crawl is approximately similar for other mid-size aircrafts. The author had taken a rough measurement of the reallife work done upon the avionics rack of Boeing 727 and on average a worker would kneel around 8 seconds to actuate works upon the avionics rack. For realism purposes, both activities or scenarios within the project were simulated or performed at the built-up mockup which is almost similar to the avionics rack of the Boeing 727.

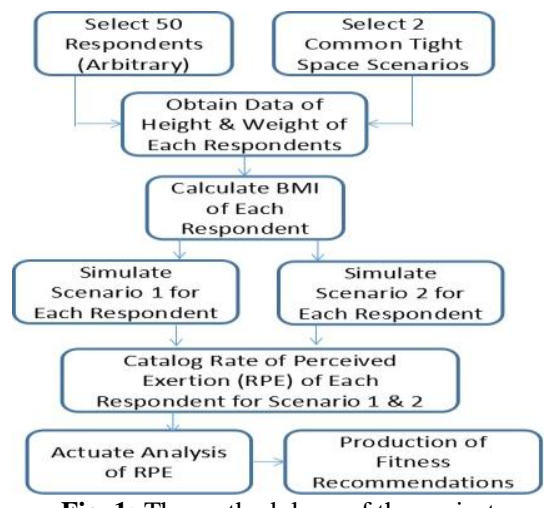

Fig. 1: The methodology of the project
The height (meter) and weight $(\mathrm{kg})$ of each respondent were measured and quantified. The weight was measured using a digital scale and the height was measured using a measuring tape. The Body Mass Index (BMI) of each respondent was calculated using equation (1) that is shown below.

$\mathrm{BMI}=$ Weight $(\mathrm{kg}) /[\operatorname{Height}(\mathrm{m}) * \operatorname{Height}(\mathrm{m})]$

Then each respondent went through Scenario 1 which was simulated for each respondent. After the end of Scenario 1, the duress that the respondents had gone through was rated by the respondents themselves. Rating was done via the usage of the scale of RPE indicated in Figure 2 (Eric Kim utilized this scale of RPE as denoted in his notes) [8]. The steps above were then actuated again for Scenario 2.

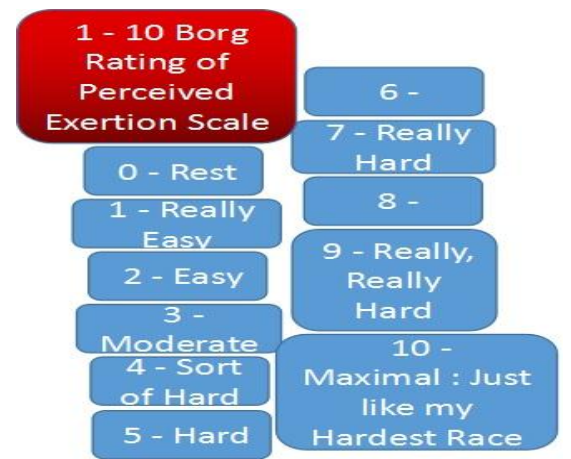

Fig. 2: The scale of RPE that was utilized

Excel spreadsheet was used to record and catalog the rated exertions of each respondent in tandem with each scenario. From these data, numerical assessments and analyses were done. The numerical assessments and analyses are:

1. Situation 1 - RPE (The Average Value)

2. Situation 2 - RPE (The Average Value)

3. Situation 1 - RPE (Trend in Graphical 2D Form)

4. Situation 2 - RPE (Trend in Graphical 2D Form)

5. Situation 1 - RPE Values (Their Frequency)

6. Situation 2 - RPE Values (Their Frequency)

7. Values of BMI of Respondents (Their Distribution)

This project also aims to decrease the RPE values of the respondents, thus advises were given in this paper pertaining to physical exercises to achieve that aim. Analysis of the RPE, literature review, and optimum practices in fitness regime were the basis for the advises given in this paper.
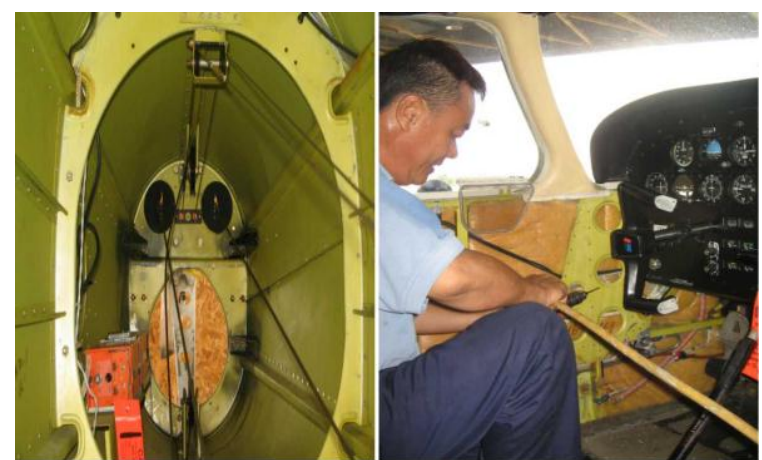

Fig. 3: Maintaining components of aircraft requires workers of aviation to crawl and kneel in spaces that are tight

\section{Results}

All the respondents (50 of them) provided their RPE values pertinent to Situation 1 and these values are shown in Figure 4 . The values of RPE among the respondents are contrasting with values ranging from high, middle, and low. Meanwhile all the respond- 
ents (50 of them) provided their RPE values pertinent to Situation 2 and these values are shown in Figure 5. For Situation 2, the RPE values among the respondents are also contrasting with values ranging from high, middle, and low. In relation to Situation 1, the average value of RPE is shown in Figure 4. In relation to Situation 2, the average value of RPE is shown in Figure 5. In relation to Situation 1, the frequency of RPE values is shown in Figure 6. In relation to Situation 2, the frequency of RPE values is shown in Figure 7. The distribution of respondents in terms of BMI values is shown in Figure 8.

RPE Situation 1 (Blue Line) \& Average Value RPE Situation 1 (Red Line)

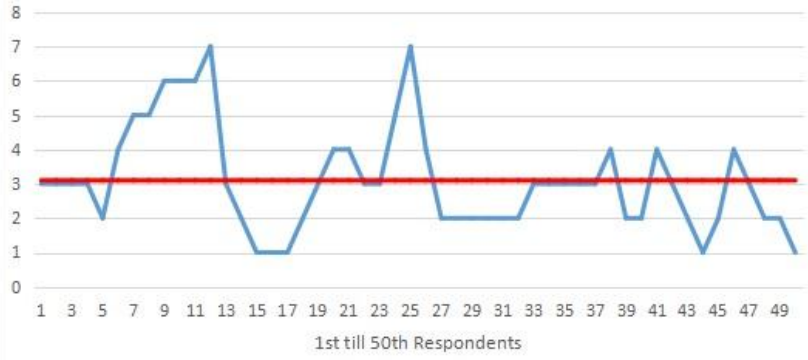

Fig. 4: RPE for Situation 1 and Average Value of RPE for Situation 1

\section{RPE Situation 2 (Blue Line) \& Average Value RPE Situation 2 (Red Line)}

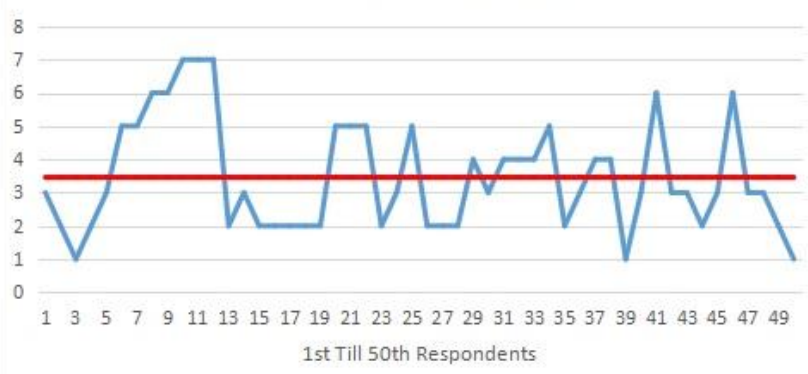

Fig. 5: RPE for Situation 2 and Average Value of RPE for Situation 2

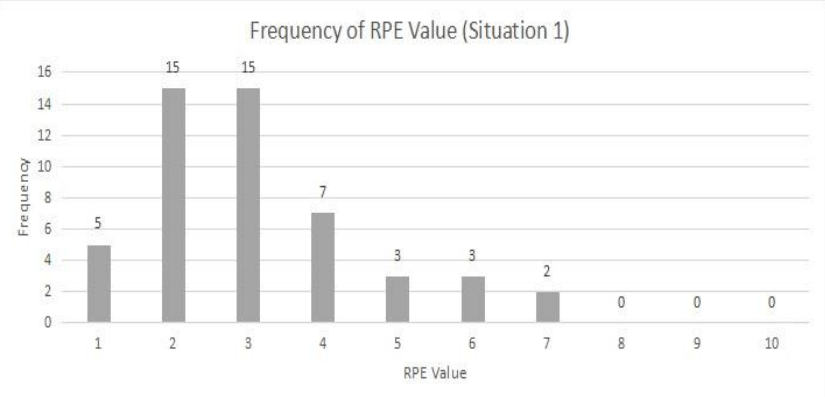

Fig. 6: Situation 1 - Values of RPE and their Frequency

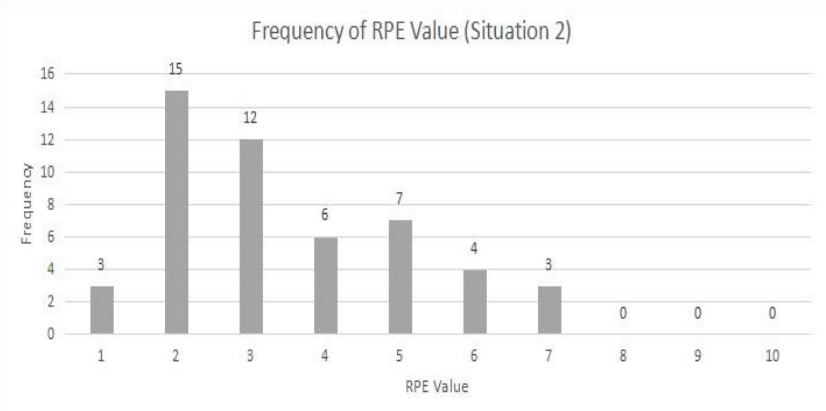

Fig. 7: Situation 2 - Values of RPE and their Frequency

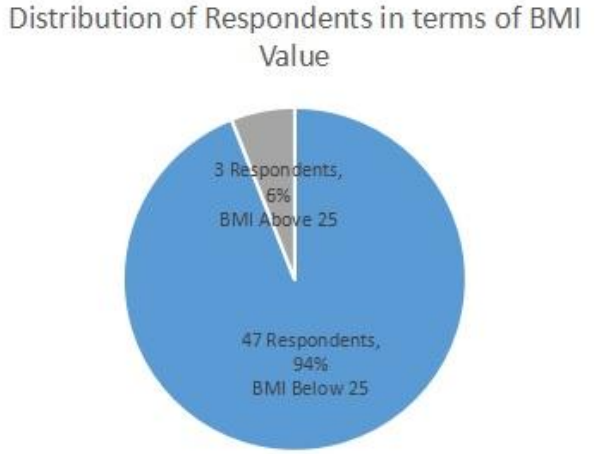

Fig. 8: Values of BMI of Respondents and Their Distribution

\section{Discussion and Results Analyses}

In relation to Figure 4, the value 7 is the highest RPE while the value 1 is the lowest RPE where this indicates numerous reactions by the respondents. Figure 6 showed that 2 respondents denoted Situation 1 as Really Hard where Situation 1 is 8 seconds of kneeling. These 2 respondents were respondent number 12 and 25 . The respondents BMI were 28.578 and 25.1559 respectively as indicated by the data sheet. Values of BMI which are over 25 are denoted as obese and overweight (male category). The respondents denoted Situation 1 as Really Hard due to perhaps their "overweight" situation where their values of BMI are over 25 which is not surprising.

Figure 6 showed that 5 respondents denoted Situation 1 as Really Easy. These respondents have values of BMI which are under 25 as indicated by the data sheet. A weight is considered healthy, in lieu with the scale of BMI, when the BMI has a value under 25 (for male). These respondents felt Really Easy pertaining to Situation 1 perhaps due to their weight which is in the healthy category. 4 till 10 would be the values of RPE which is the start of the category of hard which ranges from "sort of hard" till "maximal" as described by the scale of RPE shown in Figure 2. It is of our interest to examine the number of respondents that denotes Situation 1 within the categories of "sort of hard" until "maximal". Situation 1 was regarded hard (with the range from "sort of hard" until "maximal") by 15 respondents and this is shown in Figure 6. 15 respondents are equivalent to $30 \%$ of the whole population of respondents. It is of our interest to figure out the BMI of the 15 respondents, to observe whether the values of BMI is over 25 or less. Values of BMI over 25 occurred to 3 respondents only from the 15 respondents stated earlier as delineated by the excel sheet. The other 12 have BMI below 25 which is considered healthy but they still perceived Situation 1 as hard. This could be because at that "teenage" age they have not developed the muscle mass yet at certain body parts and hence actuating the kneeling process for the predetermined period of 8 seconds seems arduous and strainful for them.

Values of RPE, starting from 0 (rest) until 3 (moderate) are within the realm of "easy" as indicated by scale of RPE in Figure 2. "Rest" until "moderate" level of RPE for Situation 1 and the number of respondents perceiving this level is of our interest. Easy (rest until moderate) was perceived by 35 respondents in lieu with Situation 1 where this is shown in Figure 6. This figure is $70 \%$ from the whole respondents. Its of our interest to peer the BMI of the 35 respondents (whether its under 25 or contrary to it). BMI values of under 25 are obtained by the 35 respondents mentioned earlier as indicated by the excel sheet. Having healthy BMI is an advantage as it lessens the strain when conducting or going through Situation 1.

Looking at Figure 5, RPE values given by respondents range from 1 (lowest) till 7 (highest). Peering Figure 7, 3 respondents had denoted Situation 2 as Really Hard where Situation 2 is crawling for 2 meters. These 3 respondents were respondent number 10, 11, 
and 12 respectively. The 3 respondents have BMI of 22.9481 , 22.6474 , and 28.578 respectively as shown by the data sheet. Obese and overweight are denoted for a male that has a BMI value of 25 or above in accordance to the scale of BMI. Really Hard was felt by respondent number 12 pertaining to Situation 2 where his BMI is over 25 which is in the category of "overweight". Respondents number 10 and 11 , even though have BMI below 25 which is healthy, still felt the strain while actuating Situation 2. This is perhaps due to the fact that their ages are young (teenage years) and thus have not developed the necessary muscles to perform Situation 2.

Figure 7 indicated that 3 respondents felt Situation 2 as Really Easy. The 3 respondents have BMI of values 19.8347, 21.9713, and 20.984 respectively as indicated by the sheet of data. Weight of an individual is healthy when the individual has a BMI under 25 (for male) as indicated by the scale of BMI. Situation 2 was denoted as Really Easy by the respondents due to perhaps of their healthy weight.

The range of RPE from 4 (sort of hard) until 10 (maximal) falls under the "hard" category as indicated by the scale of RPE in Figure 2. It is of our interest to gain the numerical value of respondents that indicate "sort of hard" till "maximal" for Situation 2. The hard category (sort of hard until maximal) had been perceived by 20 respondents in lieu with Situation 2 as shown in Figure 7. This is $40 \%$ from the whole respondents. Values of BMI of these respondents (numbering 20) is of our interest in lieu whether these BMI values are over 25 . Values of BMI over 25 are possessed by two respondents as indicated by the sheet of excel. This means that 18 respondents have healthy BMI (BMI below 25). Even with healthy BMI, these 18 respondents felt Situation 2 as hard which is perhaps due to their "teenage" age where their muscle masses are not fully developed yet hence creating strain when they actuated Situation 2.

Values of RPE starting from 0 (rest) until 3 (moderate) are considered to be in the category of "easy" as indicated by the scale of RPE in Figure 2. It is of our interest to figure out the number of respondents that assess Situation 2 within the range of "rest" until "moderate". Situation 2 was indicated easy (rest until moderate) by 30 respondents as shown in Figure 7 . This correspond to $60 \%$ from the whole number of respondents. It is of our interest to assess the 30 respondents in terms of their BMI values (having values under 25 or otherwise). Values of BMI under 25 are possessed by 29 out of the 30 respondents mentioned earlier as indicated by the sheet of excel and only 1 respondent has BMI above 25 .

In comparison, it is shown that Situation 2 is little difficult than Situation 1 pertaining to how the respondents felt as $40 \%$ from the total respondents dictated that Situation 2 is hard while only $30 \%$ from the total respondents dictated that Situation 1 is hard. The RPE value of 3.1 is the average value for Situation 1 where this value is in the moderate category. This tells us that conducive environment exists in Situation 1 (although just seemingly) and those that deemed it difficult are advice to perform the given physical regime in order to decrease their duress in lieu with Situation 1.

The RPE value of 3.46 is the average value for Situation 2 where this value is in the moderate category. This tells us that conducive environment exists in Situation 2 (although just seemingly) and those that deemed it difficult are advice to perform the given physical regime in order to decrease their duress in lieu with Situation 2.

From the total respondents of 50 , only $6 \%$ of them have unhealthy BMI which is really good since this shows that a majority of them (94\%) have healthy BMI. This is a positive trait since maintenance students need to have healthy bodies but peering at the results showed that even with healthy BMI a good number of them felt strain while actuating Situations 1 and 2 . As highlighted earlier, these high school students need to add up more muscles in order to withstand the working conditions of Situations 1 and 2. Thus actuating the recommended physical regime would increase their muscle mass and in the same time retain or reduce their BMI to a range which is healthy.

But overall the outcome is still positive as most respondents denoted easy for Situation 1 (70\% of respondents) and Situation 2 (60\% of respondents) where this is favourable for outputs of students/workers since ecosystems that are not stressful would lead to results which are optimum.

\section{Recommended Physical Regime}

For Situations 1 and 2, the knees are used extensively where in Situation 1, which is kneeling for 8 seconds, the knee caps are pressed onto the floor for a stipulated time frame while in Situation 2, which is crawling for a distance of 2 meters, the knees are transversed at a predetermined distance. These situations or scenarios require the individuals to have rigid and strong knees and numerous physical regimes are available to improve the strength and composition of the knees. Before going through the knees exercise, we would like to advise, although its not an obligation, to firstly decrease the weight if the BMI is over 25 (based upon the data collected and analysis of the result) as a "heavy" person would likely to injure his or her knees while actuating the knees exercise. Those with surplus of weight would produce forces which are high in values upon their knees, thus creating strains upon their knees.

Decreasing the BMI of an individual is done via the actuation of High Intensity Interval Training (HIIT) (other methods are available as well). In HIIT, the individual would actuate high intense workout for a short period of time. The individual would then take a short rest and then continue with the high intense workout. This cycle is implemented several times where the accumulated duration is normally less than 30 minutes. Stoppani had laid out a typical HIIT program which is shown below [9].

1. For a duration of 15 seconds, actuate intense sprint of high mode with $100 \%$ effort

2. For a duration of 60 seconds, walk or rest

3. 10 times should the above cycle be repeated

4. For a duration of 15 seconds, actuate last intense sprint of high mode with $100 \%$ effort

5. 14 minutes is the total duration of the program above

Depending upon the outcome of the program or the objective of the individual, the program above is usually actuated for a duration of 2 till 3 weeks. The program is usually extended till the $4^{\text {th }}$ week or more if weight loss has not been achieved. The program is designed so that it would take into account other factors and thus it is not rigid.

Individuals that have BMI values of below 25 should then proceed with the knees exercise. Also, those that had actuated HIIT and achieved the value of below 25 in their BMI are encourage to actuate the exercise of the knees as well. Fundamental exercises of the knees are shown in the corresponding figures which enhance the strength of the muscle around the knee joints and thus lessen the stress upon the knees [10].

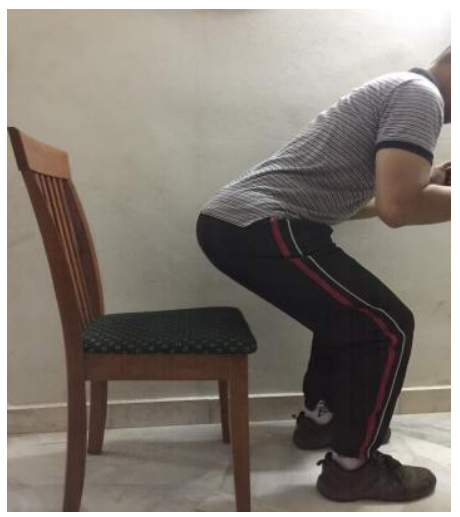

Fig. 9: Partial squats 
By looking at Figure 9, actuating partial squat is done by putting the toes in a forward pointing position and the individual's feet is set apart about hip-width while standing a distance approximately 12 inches from the front chair. The individual should then lower itself halfway to the chair while bending at the hips. All the while the abs should be tightened, and the knees should always be positioned behind the toes. Then the individual should lift him or herself up again. The individual should perform this 10 till 12 times (repetitions) and within 3 till 4 sets where a rest period of approximately 2 till 4 minutes is inserted between set. Performing this exercise 2 or 3 times a week is advisable.

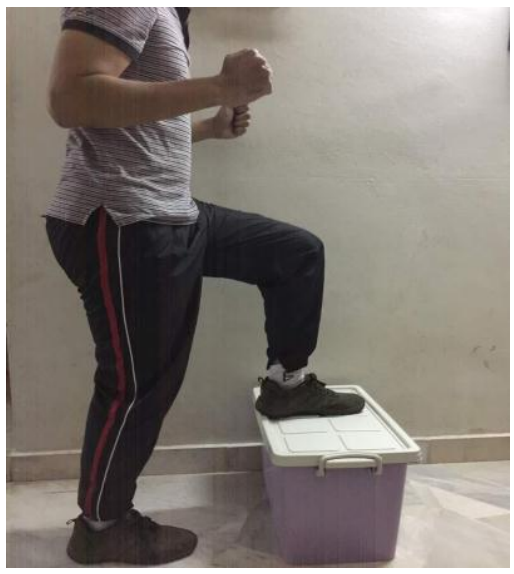

Fig. 10: Step-Ups

Figure 10 shows another knee exercise which is known as StepUps. The individual left foot is put upon or step upon the Aerobic Step Bench or staircase or a box. Then the right foot of the individual is placed momentarily on the step (top part) and lowered down to the floor. The knee should be directly over the ankle as the individual steps up. The process is then repeated or actuated for the right foot. This exercise is done 10 till 12 times (repetitions) and within 3 till 4 sets where there is a rest of approximately 2 till 4 minutes in between set. Actuating this exercise 2 or 3 times a week is advisable.

The Calf Raises is another good exercise to enhance or strengthen the knees. This is shown in Figure 11. For balance, a wall or chair could be used, and the individual would stand where his or her feet is about a hip width apart and his or her toes are pointed straight ahead. The individual would then lift slowly his or her heels off the floor until he or she rises onto the toes. This position is then hold for couple of seconds and the feet is lower back to the floor. This exercise is actuated 10 till 12 times (repetitions) and 3 till 4 sets where there is a rest of around 2 till 4 minutes between set. Actuating this exercise 2 or 3 times a week is advisable.

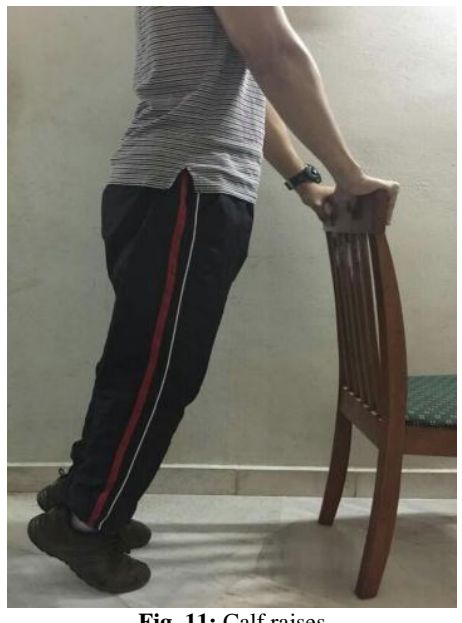

Fig. 11: Calf raises

\section{Conclusion}

It is gravely important for students or personnel within the aviation industry to be fit since their fitness would affect the work they produce. Those who are not fit are likely to produce work of lesser quality which is in contrast to those who are fit. As shown in the data collected, students or respondents (who aspire to be in Avionics field) that have healthy BMI felt less strain or exertion during their works thus they felt "comfortable" actuating their tasks. This in turn ensures that they produce optimum results in their work and hence the elevation of safety.

Peering through the completed analysis, it is shown that only slight percentage $(30 \% \& 40 \%)$ of the students (respondents) had an arduous or difficult moment completing the simulated Avionics maintenance chores. And data showed that there are cases where in lieu with the BMI that is not healthy of these individuals, the percentage mentioned encountered difficult moments. But what's interesting is that due to their "teenage" age, they have not fully developed their muscles and as stated earlier this contributed to them not being able to withstand certain working conditions.

In this paper we had laid out several related physical exercises that could be performed by the students in order for them to gain fitness or retain their fitness. For those with unacceptable level of BMI, these exercises would also lower their BMI to "healthy" level. These exercises were prescribed in relation to the data collected which indicates the exercises should focus more upon the area of the knees and thus we had laid out physical regimes which build muscles upon the area of the knees. In a nutshell, the situation is not dire as a majority of the students or respondents (70\% and $60 \%$ pertaining to Situation 1 and 2 respectively) had an easy time performing the simulated Avionics maintenance tasks.

In the opinion of the author, the fitness of the students (aspiring to be in the Avionics field) could be improved in order for them to produce optimum works during their future aviation studies and hopefully the exercise regime that was laid out could aid them in their fitness. The author has a plan to follow up with their future aviation studies and observe their future works in relation to their fitness. This hence could be a longitudinal study which would add more knowledge to the aviation industry.

\section{References}

[1] Federal Aviation Administration (2011). Aviation Maintenance Technician Handbook - General. Chapter 14, Addendum Human Factors, page 28 of Chapter 14.

[2] Federal Aviation Administration (2011). Aviation Maintenance Technician Handbook - General. Chapter 14, Addendum Human Factors, page 23 of Chapter 14.

[3] US Health Department (2015). Perceived Exertion (Borg Rating of Perceived Exertion Scale). Centers for Disease Control \& Prevention. Atlanta, Georgia.

[4] Borg, G. (1982). Psychophysical Bases of Perceived Exertion. Medicine and Science in Sports and Exercise. Volume 14, No. 5 , page 377. Stockholm Sweden.

[5] Kane, W. (1988). Stress and Aircraft Maintenance Performance in a Combat Environment. Logistics and Human Factor Division. Wright Patterson Air Force Base, Ohio. 45433-6503. August 1988.

[6] Clark, P. (2015). Overcoming Gender Barriers in Aircraft Maintenance: Women's Perceptions in the United States. Collegiate Aviation Review. Fall 2015, Volume 33, Number 2. University Aviation Association.

[7] Continental Airlines (1980). Electrical Avionic Systems Boeing 727. Book 1. Maintenance Training Department. Houston.

[8] Kim, E. (2017). Math of Fitness. Wellness Resources. Andrews University, Berrien Springs, MI.

[9] Stoppani, J. (2016). The Ultimate 8 Week HIIT for Fat Burning Program. BodyBuilding.com, Boise Idaho, July $24^{\text {th }} 2016$.

[10] Stanten, M. (2015). The Best and Worst Exercises for Bad Knees. Prevention Magazine. July $30^{\text {th }}$ 2015, Rodale Incorporated, Harlan IA 